\title{
Initial State Radiation Measurements at BESIII
}

\author{
Martin Ripka on behalf of the BESIII collaboration ${ }^{1, \star}$ \\ ${ }^{1}$ Institut für Kernphysik, Johannes Gutenberg-Universität Mainz, Johann-Joachim-Becher Weg 45, \\ 55128 Mainz, Germany
}

\begin{abstract}
Precision measurements of the hadronic cross sections $e^{+} e^{-} \rightarrow \pi^{+} \pi^{-}, \pi^{+} \pi^{-} \pi^{0}$, $\pi^{+} \pi^{-} 2 \pi^{0}, \omega \pi^{0}$ and $\pi^{+} \pi^{-} 3 \pi^{0}$ are performed at the BESIII experiment located in Beijing, China. These cross section measurements are needed as input for the Standard Model prediction of the anomalous magnetic moment of the muon, $(g-2)_{\mu}$. The initial state radiation method is used to access the energy regions of interest for $(g-2)_{\mu}$. Our results will improve the precision of the Standard Model prediction of $(g-2)_{\mu}$.
\end{abstract}

\section{Introduction}

The anomalous magnetic moment of the muon has been measured several times. The E821 experiment at BNL [1] provided the latest and most precise measurement. The experimentally measurement value and the Standard Model prediction $[2,3]$ differ by $a_{\mu}^{\text {theo }}-a_{\mu}^{\text {exp }}=(268 \pm 76) \cdot 10^{-11}$. This discrepancy corresponds to a significance of 3.5 standard deviations. More efforts are needed, both on the theory and the experimental side to see whether this deviation vanishes, or goes beyond five standard deviations, which would give good hints where to look for physics beyond the Standard Model. On the experimental side, there are two new measurements planed. One in Japan at J-PARC [4] and another in the USA at Fermilab [5]. On the theory side, the largest contribution to the uncertainty of $a_{\mu}^{\text {theo }}$ comes from the leading-order (LO) Vacuum Polarization (VP) contribution $\mu^{\mathrm{VP}, \mathrm{LO}}$ of the QCD part of the quantum corrections, which cannot be calculated with the perturbative approach. Instead, the optical theorem can be used to relate the VP contribution to hadronic cross sections, which can be measured experimentally. The size of the contributions to $a_{\mu}^{\mathrm{VP}, \mathrm{LO}}$ of the most important hadronic cross sections are shown in Fig. 1 together with the size of their uncertainties. Three of the important hadronic cross sections that contribute to $a_{\mu}^{\mathrm{VP}, \mathrm{LO}}$ come from the processes $e^{+} e^{-} \rightarrow \pi^{+} \pi^{-}, \pi^{+} \pi^{-} \pi^{0}$ and $e^{+} e^{-} \rightarrow \pi^{+} \pi^{-} \pi^{0} \pi^{0}$, where the latter one contributes with the largest relative uncertainty.

The Initial State Radiation (ISR) technique is applied to access the cross sections at energies below $1.8 \mathrm{GeV}$, which is the region of interest for $(g-2)_{\mu}$. Therefore, an additional photon is required, which is emitted before the collision from the electron or positron. If a photon with energy $E_{I S R}$ is emitted from the initial state with center-of-mass (c.m.) energy $s$, the effective c.m. energy $s^{\prime}=m_{\text {had }}^{2}$ available for the production of the final state is reduced according to $s^{\prime}=s-2 E_{I S R} \sqrt{s}$. The desired non-radiative cross section $\sigma_{\text {had }}$ can be calculated from the ISR cross section $\frac{d \sigma_{(\text {had }+\gamma)}}{d m_{\text {had }}}$, via

$$
\frac{d \sigma_{(h a d+\gamma)}}{d m_{\text {had }}}=\frac{2 m_{\text {had }}}{s} W\left(s, E_{I S R}\right) \sigma_{\text {had }},
$$

^e-mail: ripka@uni-mainz.de 


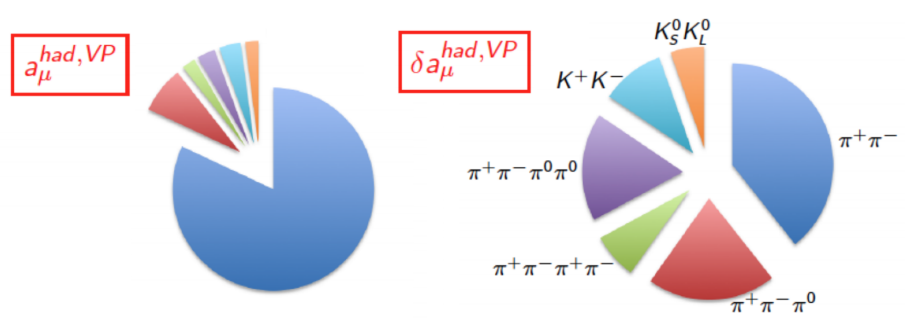

Figure 1. Contributions (left) and their uncertainties (right) of the most important hadronic cross sections, that contribute to $a_{\mu}^{\mathrm{VP}, \mathrm{LO}}$.

where $W\left(s, E_{I S R}\right)$ is the radiator function, that describes the probability of the emission of ISR photons. The ISR technique has two analysis strategies. The first one is the ISR tagged strategy, where the ISR photon is required to be detected. The second case is the ISR untagged strategy, where the ISR photon escapes detection, but its four-momentum can be reconstructed by imposing four-momentum conservation. The latter case has much higher statistics, since ISR photons are predominantly emitted at $\left|\cos \theta_{I S R}\right| \approx 1$. However, for kinematic reasons at BESIII, the detection efficiency drops to zero below $m_{\text {had }} \lesssim 1 \mathrm{GeV}$ for the untagged scenario. The tagged ISR scenario can be used to measure the cross section below $1 \mathrm{GeV}$.

BESIII is a general purpose detector, covering 93\% of the solid angle. It is operating at the $e^{+} e^{-}$ collider BEPCII. A detailed description of the facilities is given in Ref. [6]. Our measurements are based on $2.93 \mathrm{fb}^{-1}$ of data [7, 8], taken at a c.m. energy $\sqrt{s}=3.773 \mathrm{GeV}$ with the BESIII detector.

\section{$2 e^{+} e^{-} \rightarrow \pi^{+} \pi^{-}$cross section}

The analysis presented in this section is published in Ref. [8]. A deviation between the existing results of BaBar $[9,10]$ and KLOE [11-13] on the level of 3-5\% called for a third, independent high precision measurement, since both, BaBar and KLOE claimed a sub-percent precision. At BESIII we performed a new measurement applying the tagged ISR technique. The shower shape variables from the electromagnetic calorimeter are used as input parameters for an Artificial Neuronal Network (ANN) to separate muons from pions. In this way, a very clean $\pi^{+} \pi^{-} \gamma_{I S R}$ sample is obtained. Applying Eq. 1 on the number of selected events and correcting for vacuum polarization effects [14] and final state radiation effects, the $e^{+} e^{-} \rightarrow \pi^{+} \pi^{-}$cross section is calculated. The total systematic uncertainty is $0.9 \%$. The two largest contributions of uncertainty come from radiator function with $0.5 \%$ and the luminosity measurement also with $0.5 \%$. As shown the top panels of Fig. 2, the BESIII result agrees with BaBar above $\sqrt{s} \gtrsim 0.8 \mathrm{GeV}$ and with KLOE below $\sqrt{s} \lesssim 0.8 \mathrm{GeV}$. The contribution to the anomalous magnetic moment in the $\rho(770)$ resonance region of the BESIII measurement is $a_{\mu}^{\pi \pi, L O}(600-900 \mathrm{MeV})=(368.2 \pm 2.5 \pm 3.3) \cdot 10^{-10}$. A comparison of the KLOE, BaBar and BESIII results on $a_{\mu}^{\pi \pi, L O}(600-900 \mathrm{MeV})$ is illustrated in the lower panel of Fig. 2. Our measurement confirms a discrepancy of more than three standard deviations between experiment and theory of $a_{\mu}$.

\section{$3 e^{+} e^{-} \rightarrow \pi^{+} \pi^{-} \pi^{0}$ cross section}

The $e^{+} e^{-} \rightarrow \pi^{+} \pi^{-} \pi^{0}$ cross section can be measured with the BESIII measurement in the region of the $\omega$ and $\phi$ resonances using the tagged ISR technique. Above the $\phi$ resonance, both, the tagged and 


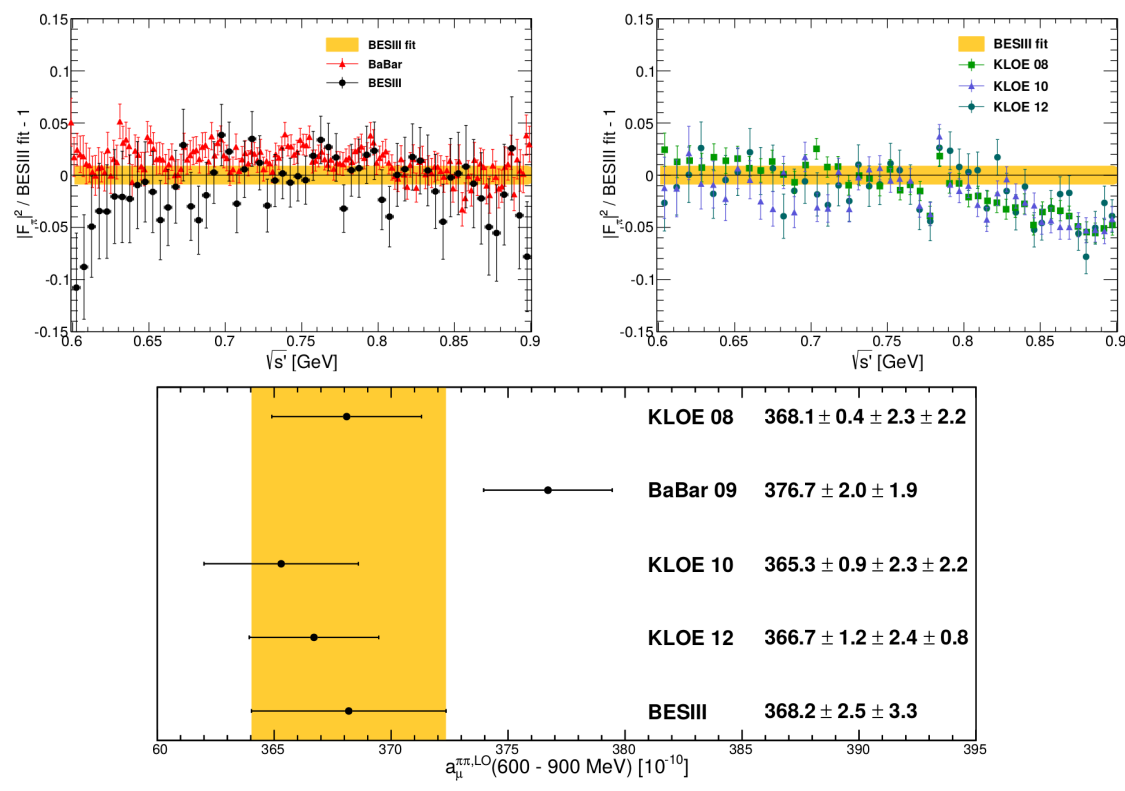

Figure 2. Comparison of the fitted pion form factor from BESIII to BaBar (top left) and KLOE (top right). Comparison of the results for $a_{\mu}^{\pi \pi, L O}(600-900 \mathrm{MeV})$ between KLOE, BaBar and BESIII (bottom). The errors bars include statistical and systematic errors.

untagged ISR methods are performed to measure the $e^{+} e^{-} \rightarrow \pi^{+} \pi^{-} \pi^{0}$ cross section. Fig. 3 shows the $e^{+} e^{-} \rightarrow \pi^{+} \pi^{-} \pi^{0}$ cross section calculated by Eq. 1 from the selected $\pi^{+} \pi^{-} \pi^{0} \gamma_{I S R}$ events in the three regions explained before. Vacuum polarization effects and final state radiation effects are corrected for. The systematic uncertainty of the BESIII measurement is $2 \%$ in the resonance regions. The BESIII result agrees with previous results from CMD2 [16, 17] and SND $[18,19]$ within errors in the $\omega$ and $\phi$ resonance regions. In the region above the $\phi$ resonance, the BESIII result clearly supports the BaBar measurement [20]. We fit of the cross section line shape with the formula from Ref. [18],
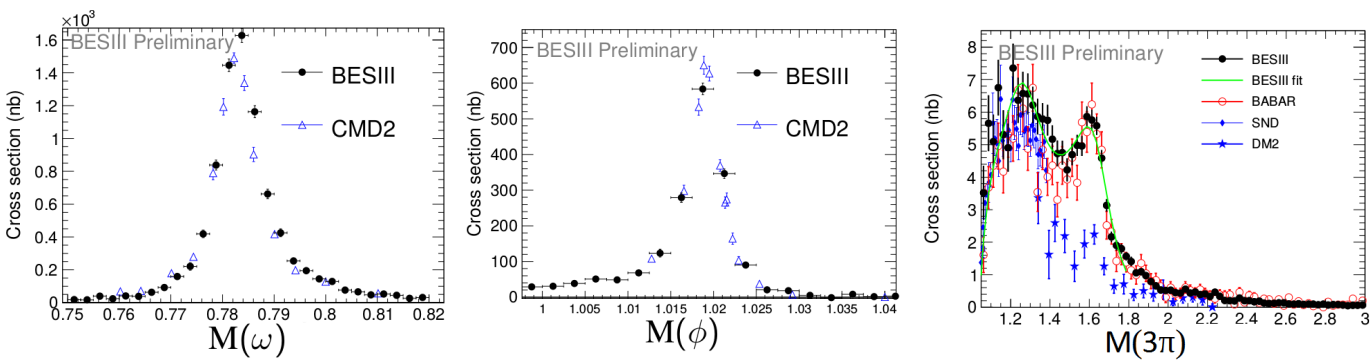

Figure 3. $e^{+} e^{-} \rightarrow \pi^{+} \pi^{-} \pi^{0}$ cross section in the $\omega$-resonance region (left), the $\phi$-resonance region (middle) and in the region above the $\phi$ resonance (right). 
Table 1. Fit results of the fit to the $\pi^{+} \pi^{-} \pi^{0}$ cross section.

\begin{tabular}{l|c|c}
\multicolumn{2}{c}{ BES Prelimingy } \\
\hline \hline Parameters & PDG & This result \\
\hline$\chi^{2} / \mathrm{NDF}$ & - & $443 / 390$ \\
\hline$m_{\omega}\left(\mathrm{MeV} / c^{2}\right)$ & $782.65 \pm 0.12$ & $783.20 \pm 0.07 \pm 0.23$ \\
$m_{\phi}\left(\mathrm{MeV} / c^{2}\right)$ & $1019.46 \pm 0.02$ & $1020.00 \pm 0.06 \pm 0.30$ \\
$m_{\omega^{\prime}}\left(\mathrm{MeV} / c^{2}\right)$ & $1400 \sim 1450$ & $1388 \pm 39 \pm 52$ \\
$m_{\omega^{\prime \prime}}\left(\mathrm{MeV} / c^{2}\right)$ & $1670 \pm 30$ & $1699 \pm 9 \pm 6$ \\
\hline$\Gamma_{\omega}\left(\mathrm{MeV} / c^{2}\right)$ & $8.49 \pm 0.08$ & $\mathrm{PDG}$ \\
$\Gamma_{\phi}(\mathrm{MeV})$ & $4.26 \pm 0.04$ & $\mathrm{PDG}$ \\
$\Gamma_{\omega^{\prime}}(\mathrm{MeV})$ & $180 \sim 250$ & $629 \pm 155 \pm 212$ \\
$\Gamma_{\omega^{\prime \prime}}(\mathrm{MeV})$ & $315 \pm 35$ & $331 \pm 40 \pm 28$ \\
\hline$\left(\mathcal{B}_{\omega \rightarrow e^{+} e^{-}} \times \mathcal{B}_{\omega \rightarrow 3 \pi}\right)\left(10^{-5}\right)$ & $6.49 \pm 0.11$ & $6.94 \pm 0.08 \pm 0.17$ \\
$\left(\mathcal{B}_{\phi \rightarrow e^{+} e^{-}} \times \mathcal{B}_{\phi \rightarrow 3 \pi}\right)\left(10^{-5}\right)$ & $4.53 \pm 0.10$ & $4.20 \pm 0.08 \pm 0.17$ \\
$\left(\mathcal{B}_{\omega^{\prime} \rightarrow e^{+} e^{-}} \times \mathcal{B}_{\omega^{\prime} \rightarrow 3 \pi}\right)\left(10^{-6}\right)$ & $0.82 \pm 0.08$ & $0.84 \pm 0.09 \pm 0.09$ \\
$\left(\mathcal{B}_{\omega^{\prime \prime} \rightarrow e^{+} e^{-}} \times \mathcal{B}_{\omega^{\prime \prime} \rightarrow 3 \pi}\right)\left(10^{-6}\right)$ & $1.30 \pm 0.20$ & $1.14 \pm 0.15 \pm 0.15$ \\
\hline \hline $\mathcal{B}_{J / \psi \rightarrow 3 \pi}(\%)$ & $2.11 \pm 0.07$ & $2.18 \pm 0.03 \pm 0.06$ \\
\hline \hline
\end{tabular}

Table 2. Preliminary results on $a_{\mu}^{\pi^{+} \pi^{-} 2 \pi^{0}, \mathrm{LO}}$ from BaBar and BESIII.

\begin{tabular}{c|c} 
& $a_{\mu}^{\pi^{+} \pi^{-} 2 \pi^{0}, \mathrm{LO}} / 10^{-10}$ \\
\hline BESIII (preliminary) & $18.6 \pm 0.3 \pm 0.6$ \\
BaBar (preliminary) & $17.9 \pm 0.1 \pm 0.6$
\end{tabular}

which contains the four resonances $\omega, \phi, \omega^{\prime}$ and $\omega^{\prime \prime}$. Our fit results are listed in Table 1 . The obtained resonance parameters agree with the PDG values [21].

\section{$4 e^{+} e^{-} \rightarrow \pi^{+} \pi^{-} 2 \pi^{0}$ cross section}

The $e^{+} e^{-} \rightarrow \pi^{+} \pi^{-} 2 \pi^{0}$ cross section is measured with both, the tagged and untagged ISR methods. The final result is obtained from the error weighted mean of both ISR methods. Irreducible background contributions from $e^{+} e^{-} \rightarrow \pi^{+} \pi^{-} 3 \pi^{0}$ and $e^{+} e^{-} \rightarrow \pi^{+} \pi^{-} 3 \pi^{0} \gamma_{\text {ISR }}$ occur in the tagged as well as the untagged method. The $e^{+} e^{-} \rightarrow \pi^{+} \pi^{-} 3 \pi^{0}$ and $e^{+} e^{-} \rightarrow \pi^{+} \pi^{-} 3 \pi^{0} \gamma_{\text {ISR }}$ processes are measured additionally, in order to correct the corresponding MC simulations. With this data driven method, the accuracy and the systematic uncertainty of the background subtraction can be significantly reduced. For the $\pi^{+} \pi^{-} 3 \pi^{0}$ channel, the HelPWA generator [? ] is used, which produces a simulation according to a partial waves analysis of data. The $\pi^{+} \pi^{-} 2 \pi^{0}$ cross section combined from the tagged and untagged methodes, shown in Fig. 4, is calculated according to Eq. 1, from the selected $\pi^{+} \pi^{-} 2 \pi^{0} \gamma_{\text {ISR }}$ events. Vacuum polarization effects and final state radiation effects are corrected for. The cross section spectrum is not unfolded for detector resolution effects, so the $J / \psi$ appears smeared with detector resolution in Fig. 4. The total systematic uncertainty of the combined measurement is $3 \%$. Our result is in agreement with previous measurements [22-29] (except ND). Moreover, we calculate the contribution to $a_{\mu}^{V P, L O}$. The result is shown in Table 2 together with the preliminary result from BaBar [30]. The results agree within errors.

Moreover, the sub-process $e^{+} e^{-} \rightarrow \omega \pi^{0}$ is investigated. Fits of the $M\left(\pi^{+} \pi^{-} \pi^{0}\right)$ distributions are performed for each $M\left(\pi^{+} \pi^{-} 2 \pi^{0}\right)$ interval. The fit function at each interval is a double Gaussian for the $\omega$ peak plus a third order polynomial for the non- $\omega$ continuum contribution. The cross section obtained from the fits is shown Fig. 4. The measured cross section is corrected for vacuum polarization effects and final state radiation effects. The systematic uncertainty varies between $4-5 \%$. The result is in good agreement with previous experiments [23, 24, 29, 31-34]. 

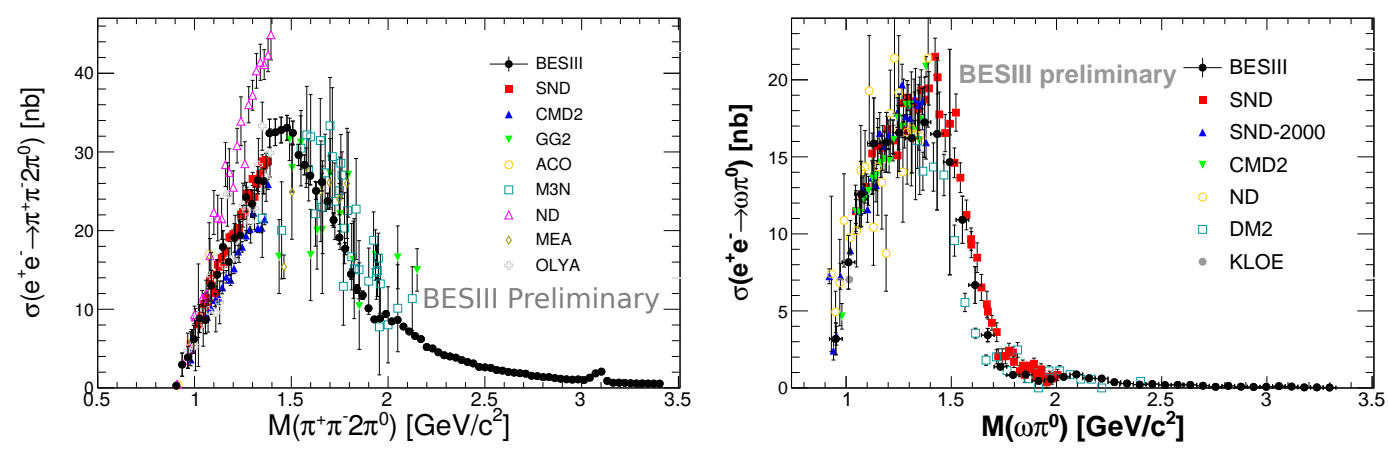

Figure 4. $\pi^{+} \pi^{-} 2 \pi^{0}$ cross section (left) and $\omega \pi^{0}$ cross section (right).

\section{$5 e^{+} e^{-} \rightarrow \pi^{+} \pi^{-} 3 \pi^{0}$ cross section}

The process $e^{+} e^{-} \rightarrow \pi^{+} \pi^{-} 3 \pi^{0} \gamma_{\mathrm{ISR}}$ and its sub-processes $e^{+} e^{-} \rightarrow \omega 2 \pi^{0} \gamma_{I S R}$ and $e^{+} e^{-} \rightarrow \eta \pi^{+} \pi^{-} \gamma_{I S R}$ are measured to validate and improve the MC description of the background contributions to the $e^{+} e^{-} \rightarrow \pi^{+} \pi^{-} 2 \pi^{0}$ cross section. In fact, the cross sections of $e^{+} e^{-} \rightarrow \pi^{+} \pi^{-} 3 \pi^{0}, e^{+} e^{-} \rightarrow \eta \pi^{+} \pi^{-}$and $e^{+} e^{-} \rightarrow \omega 2 \pi^{0}$ can be also extracted from this measurement. The resulting cross sections, corrected for vacuum polarization effects and final state radiation effects, are shown in Fig. 5. Again, the cross section spectrum is not unfolded for detector resolution, so the $J / \psi$ appears smeared with detector resolution in Fig. 5.

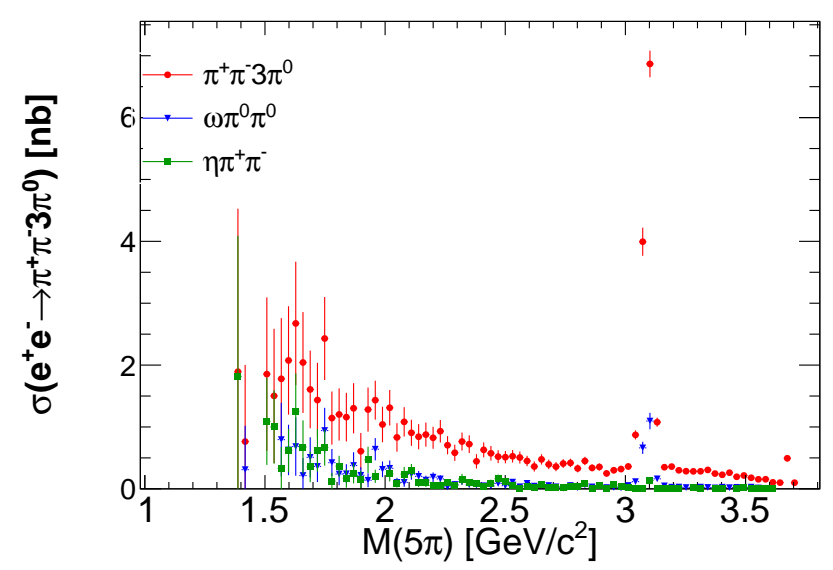

Figure 5. Cross section of $\pi^{+} \pi^{-} 3 \pi^{0}, \omega \pi^{0} \pi^{0}$ and $\eta \pi^{+} \pi^{-}$.

\section{Summary}

We have performed new measurements of the cross sections $\sigma\left(e^{+} e^{-} \rightarrow \pi^{+} \pi^{-}\right), \sigma\left(e^{+} e^{-} \rightarrow \pi^{+} \pi^{-} \pi^{0}\right)$, $\sigma\left(e^{+} e^{-} \rightarrow \pi^{+} \pi^{-} 2 \pi^{0}\right), \sigma\left(e^{+} e^{-} \rightarrow \omega \pi^{0}\right), \sigma\left(e^{+} e^{-} \rightarrow \pi^{+} \pi^{-} 3 \pi^{0}\right), \sigma\left(e^{+} e^{-} \rightarrow \omega \pi^{0} \pi^{0}\right)$ and,$\sigma\left(e^{+} e^{-} \rightarrow\right.$ 
$\eta \pi^{+} \pi^{-}$) applying the tagged and untagged ISR methods using $2.93 \mathrm{fb}^{-1}$ of data, taken at a centerof-mass energy $\sqrt{s}=3.773 \mathrm{GeV}$ with the BESIII detector. Our results agree with previous experiments and compete in precision with the recent BaBar measurements. Moreover, we find $a_{\mu}^{\pi \pi, L O}=(368.2 \pm 2.5 \pm 3.3) \cdot 10^{-10}$ and $a_{\mu}^{\pi^{+} \pi^{-} 2 \pi^{0}, \mathrm{LO}}=(18.6 \pm 0.3 \pm 0.6) \cdot 10^{-10}$ for the leading-order vacuum polarization parts of the anomalous magnetic momentum of the muon.

\section{References}

[1] G. W. Bennett et al. [Muon G-2 Collaboration], Phys. Rev. D 73072003 (2006).

[2] M. Davier, A. Hoecker, B. Malaescu and Z. Zhang, arXiv:1706.09436 [hep-ph].

[3] K. Hagiwara, A. Keshavarzi, A. D. Martin, D. Nomura and T. Teubner, Nucl. Part. Phys. Proc. 287-288, 33 (2017).

[4] T. Mibe [J-PARC g-2 Collaboration], Nucl. Phys. Proc. Suppl. 218, 242 (2011).

[5] B. L. Roberts, Chin. Phys. C 34, 741 (2010).

[6] M. Ablikim et al. [BESIII Collaboration], Nucl. Instrum. Meth. A 614345 (2010).

[7] M. Ablikim [BESIII Collaboration], Chin. Phys. C 37123001 (2013).

[8] M. Ablikim et al., Phys. Lett. B 753, 629 (2016).

[9] B. Aubert et al., [BaBar Collaboration], Phys. Rev. Lett. 103, 231801 (2009).

[10] J. P. Lees et al., [BaBar Collaboration], Phys. Rev. D 86, 032013 (2012).

[11] F. Ambrosino et al., [KLOE Collaboration], Phys. Lett. B 670, 285 (2009).

[12] F. Ambrosino et al., [KLOE Collaboration], Phys. Lett. B 700, 102-110 (2011).

[13] D. Babusci et al., [KLOE Collaboration], Phys. Lett. B 720, 336-343 (2013).

[14] S. Actis et al. [Working Group on Radiative Corrections and Monte Carlo Generators for Low Energies Collaboration], [arXiv:0912.0749 [hep-ph]].

F. Ignatov, http://cmd.inp.nsk.su/ ignatov/vpl.

[15] H. Czyż, M. Gunia and J. H. Kühn, JHEP 1308, 110 (2013)

[16] R. R. Akhmetshin et al. [CMD-2 Collaboration], Phys. Lett. B 476, 33 (2000).

[17] R. R. Akhmetshin et al., Phys. Lett. B 642, 203 (2006).

[18] M. N. Achasov et al., Phys. Rev. D 68, 052006 (2003).

[19] M. N. Achasov et al., Phys. Rev. D 66, 032001 (2002).

[20] B. Aubert et al. [BaBar Collaboration], Phys. Rev. D 70, 072004 (2004).

[21] C. Patrignani et al. [Particle Data Group], Chin. Phys. C 40, no. 10, 100001 (2016).

[22] L. M. Kurdadze et al., J. Exp. Theor. Phys. Lett. 43643 (1986).

[23] R. R. Akhmetshin et al. [CMD-2 Collaboration], Phys. Lett. B 466, 392 (1999).

[24] S. I. Dolinsky et al., Phys. Rept. 202, 99 (1991).

[25] M. N. Achasov et al., Preprint BUDKER-INP-2001-34 (Novosibirsk, 2001).

[26] G. Cosme et al., Phys. Lett. B 63, 349 (1976).

[27] G. Cosme et al., Nucl. Phys. B 152215 (1979).

[28] B. Esposito et al., Lett. Nuovo Cim. 31, 445 (1981).

[29] C. Bacci et al., Nucl. Phys. B 184, 31 (1981).

[30] K. Griessinger [BaBar Collaboration], Nucl. Part. Phys. Proc. 287-288, 47 (2017).

[31] D. Bisello et al. [DM2 Collaboration], Preprint LAL-90-35 (Orsay, 1990).

[32] M. N. Achasov et al., Phys. Lett. B 486, 29 (2000).

[33] M. N. Achasov et al., arXiv:1610.00235 [hep-ex].

[34] F. Ambrosino et al. [KLOE Collaboration], Phys. Lett. B 669, 223 (2008). 